\title{
Brillouin Frequency Shift estimation in BOTDA via subpixel processing
}

\author{
R. Ruiz-Lombera*, J. Mirapeix, I. Laarossi, L. Rodríguez-Cobo, J.M. Lopez-Higuera \\ Photonics Engineering Group, University of Cantabria, Plaza de la Ciencia s/n, Edificio I+D Teleco, \\ 39005, Santander (Spain)
}

\begin{abstract}
In this paper we propose the employment of sub-pixel algorithms for the estimation of the central frequency of the Brillouin Gain Spectrum in a Brillouin Optical Time Domain Analyzer. The experimental results will show that the proposed solution shows a good performance when the chosen frequency step for the required frequency sweep is high. If the improved computational efficiency in comparison to the traditional Lorentzian fitting is also considered, it can be concluded that this approach may be of great interest for dynamic measurement scenarios.
\end{abstract}

Keywords: stimulated Brillouin scattering, distributed sensing, BOTDA, Brillouin frequency shift, sub-pixel algorithm, Lorentzian fitting

\section{INTRODUCTION}

Distributed sensing based on the stimulated Brillouin scattering (SBS) has been an intensive area of research during the last years with applications from the energy to the civil engineering sectors, just to mention some examples. SBS is based on the employment of two counter-propagating waves, the so-called pump and probe that interact via acoustic phonons within the optical fiber to give rise to an amplified probe signal. Its frequency is directly related to the strain and temperature at each point of the optical fiber, provided that the pump wave is pulsed.

Although there are different schemes based on the frequency [1] and correlation [2] domains, the most common implementation of SBS-based distributed sensing is the Brillouin Optical Time Domain Analysis (BOTDA), usually by means of the sideband technique [3]. In this configuration the frequency of the pulsed pump is normally kept constant, while the probe signal, obtained via an electro-optical modulator (EOM) and a RF generator, is swept in frequency to allow a reconstruction of the Brillouin Gain Spectrum (BGS) at the detector. Afterwards, the Brillouin Frequency Shift (BFS) is estimated as the central frequency of the BGS by using a Lorentzian fitting process.

It is worth mentioning that this frequency sweep, in addition to the required averaging of the acquired traces, implies a significant increase in the measurement time over the whole fiber under test (FUT), thus avoiding dynamic measurements in the so-called standard BOTDA implementations. Different variations have been proposed in this regard to allow fast measurements, as the slope-assisted BOTDA [4], the employment of multiple pump and probe waves to avoid the frequency sweep [5] or the use of the Brillouin phase-shift and RF demodulation [6].

Although there are other factors limiting the measurement time, as the time of flight of the pump pulses, the frequency scanning is probably the most relevant. In addition, the above mentioned fitting process used for the BFS estimation is also time consuming, as it involves an iterative stage. In this paper we explore the employment of subpixel algorithms instead of the Lorentzian fitting. These algorithms have been extensively used for image processing [7], but they have been also applied in fiber Bragg grating (FBG) applications [8] or plasma optical spectroscopy for welding diagnostics [9]. Their computational performance as well as the resulting accuracy for different scanning granularities will be analyzed for distributed BOTDA measurements over $50 \mathrm{~km}$.

*ruben.ruiz@unican.es; phone (+34) 942 200877; fax (+34) 942200877; http://gif.teisa.unican.es/

Sixth European Workshop on Optical Fibre Sensors, edited by Elfed Lewis, Proc. of SPIE Vol. 9916 , 99162L · (c) 2016 SPIE · CCC code: 0277-786X/16/\$18 · doi: 10.1117/12.2236862 


\section{EXPERIMENTAL ISSUES}

The conventional BOTDA scheme depicted in Fig. 1 was used to perform the required experimental tests. A single laser source $(\lambda=1550.92 \mathrm{~nm})$ generates the light required for both pump and probe waves. The optical coupler located at the output of the laser source divides the light (10\% to the pump wave, $90 \%$ to the probe wave) into two branches. The upper one generates the pump pulses via a semiconductor optical amplifier (SOA). The pump pulse is amplified via an erbium doped fiber amplifier (EDFA) and then a polarization scrambler is employed to avoid the polarization dependence of the SBS gain along the fiber. The probe wave is generated by an EOM and RF generator, thus giving rise to two sidebands and a carrier that is suppressed by adjusting the bias voltage of the EOM. Both sidebands are transmitted via an optical isolator to the FUT, where they will interact with the pump pulse. The detection stage is formed by two optical circulators, which allow to select the lower frequency sideband with a FBG, and a high-transimpedance gain $125 \mathrm{MHz}$ photodetector and acquisition card (ACQ) integrated in a PC.

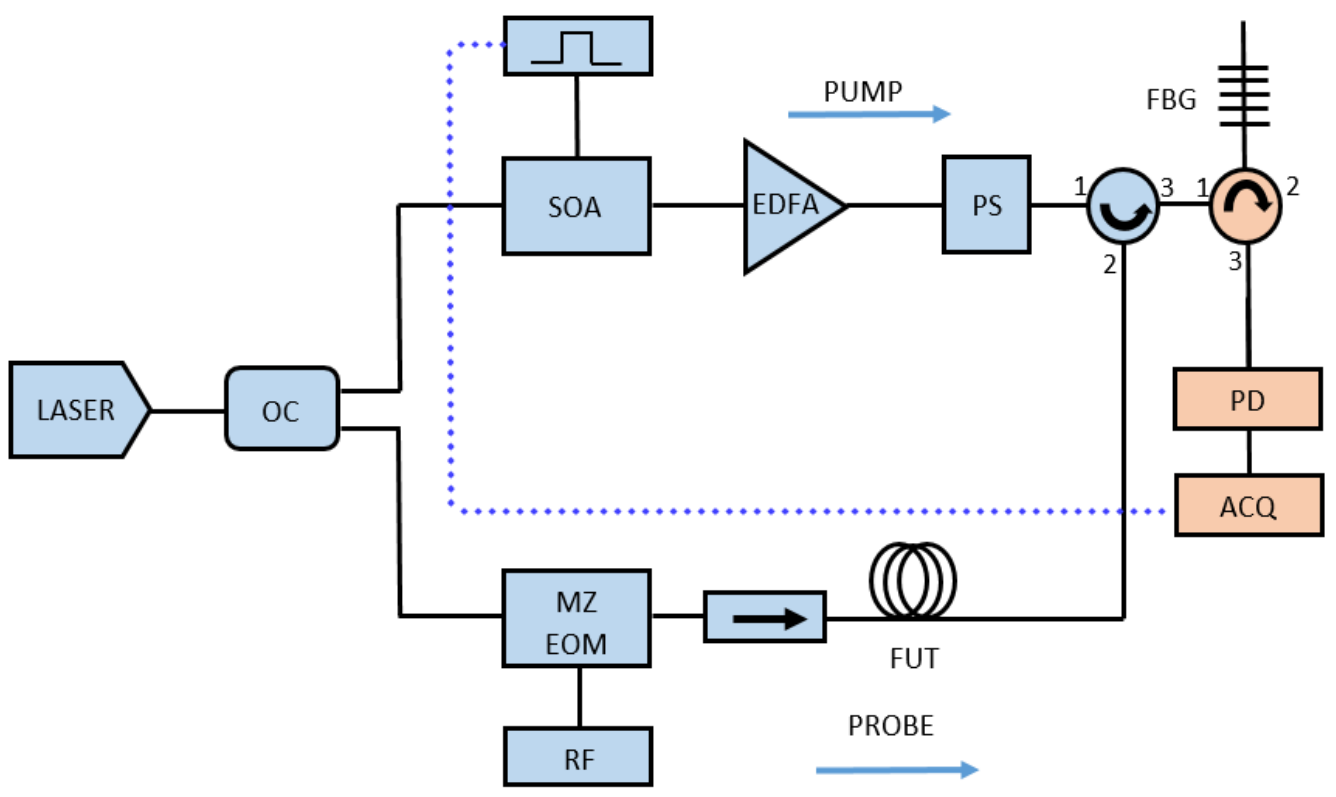

Figure 1. Schematic setup of the BOTDA system used in the experimental tests: Optical Coupler (OC), Semiconductor Optical Amplifier (SOA), Erbium Doped Fiber Amplifier (EDFA), Polarization Scrambler (PS), Match-Zender ElectroOptical Modulator (MZ-EOM), RF Generator (RF), Fiber Under Test (FUT), Fiber Bragg Grating (FBG), Photodetector (PD) and Acquisition Card (ACQ).

To explore the proposed solution the centroid subpixel algorithm CDA was selected. It is based on the estimation of the mass centre of the peak under analysis:

$$
f_{s u b}=\frac{\sum_{j} f_{j} i_{j}}{\sum_{j} i_{j}},
$$

where $f_{\text {sub }}$ is the subpixel estimation of the peak frequency and $f_{j}$ and $i_{j}$ the frequency and intensity associated with the pixel $j$. The code to perform the BFS estimation was implemented in Matlab, and compared to the conventional Lorentzian fitting.

To evaluate their corresponding performances a $50 \mathrm{~km}$ standard monomode fiber was deployed with an estimated BFS of approximately $10.70 \mathrm{GHz}$. Several measurements were performed considering a frequency range between 10.59 and $10.79 \mathrm{GHz}$, and scanning granularities of 2, 4, 6, 8, 10 and $20 \mathrm{MHz}$. A 4096 averaging of the acquired BOTDA traces was performed to improve the signal-to-noise ratio (SNR). 
Figure 2 (a) depicts BFS profiles derived of employing Lorentzian fitting and different scanning granularities: $2 \mathrm{MHz}$ (black), $10 \mathrm{MHz}$ (red) and $20 \mathrm{MHz}$ (blue). As expected, the use of a smaller granularity gives rise to a less noisy profile, although the differences between the 2 and $10 \mathrm{MHz}$ profiles are subtle. The BFS curve obtained with the $20 \mathrm{MHz}$ granularity exhibits a clearly degraded SNR, as well as an error in the BFS estimation that increases with distance. For example, the standard deviation of the $2 \mathrm{MHz}$ granularity BFS profile between 30 and $40 \mathrm{~km}$ is $1.33 \mathrm{MHz}$, while the one associated with the $20 \mathrm{MHz}$ granularity is $1.69 \mathrm{MHz}$. The corresponding mean BFS values are 10.691 and $10.693 \mathrm{GHz}$.

Figure 2(b) presents the comparison of the worst case BFS profile (for a $20 \mathrm{MHz}$ granularity) obtained via Lorentzian fitting and the result of substituting it with the CDA subpixel processing. It can be observed how the SNR clearly improves with the proposed solution, with a standard deviation, again evaluated within the 30 to $40 \mathrm{~km}$ section, of 0.86 $\mathrm{MHz}$. The root mean square error over the whole FUT, computed using the $2 \mathrm{MHz}$ granularity profile as a reference, is $2.68 \mathrm{MHz}$ for the Lorentzian fitting and $1.80 \mathrm{MHz}$ using CDA. It should be mentioned that the saturated profile obtained for the $20 \mathrm{MHz}$ granularity (between $\mathrm{x} \approx 10$ and $20 \mathrm{~km}$ ) and Lorentzian fitting is precisely due to the chosen granularity and not to other effects to be found in BOTDA traces such as modulation instability.

Apart from these results, the main motivation of avoiding the conventional fitting process might lie in its high computational cost. The average processing time (over 10 tests) of the CDA processing was 0.241 s for the whole BFS of $2 \mathrm{MHz}$ granularity, considering a data matrix of 101 (frequency) x 12000 (spatial) samples. In the same scenario, the Lorentzian fitting gave rise to a processing time in the order of several minutes. These performance tests were carried out using MATLAB in a conventional laptop (i5-2410M (2.30 GHz) processor with 4 GB RAM).
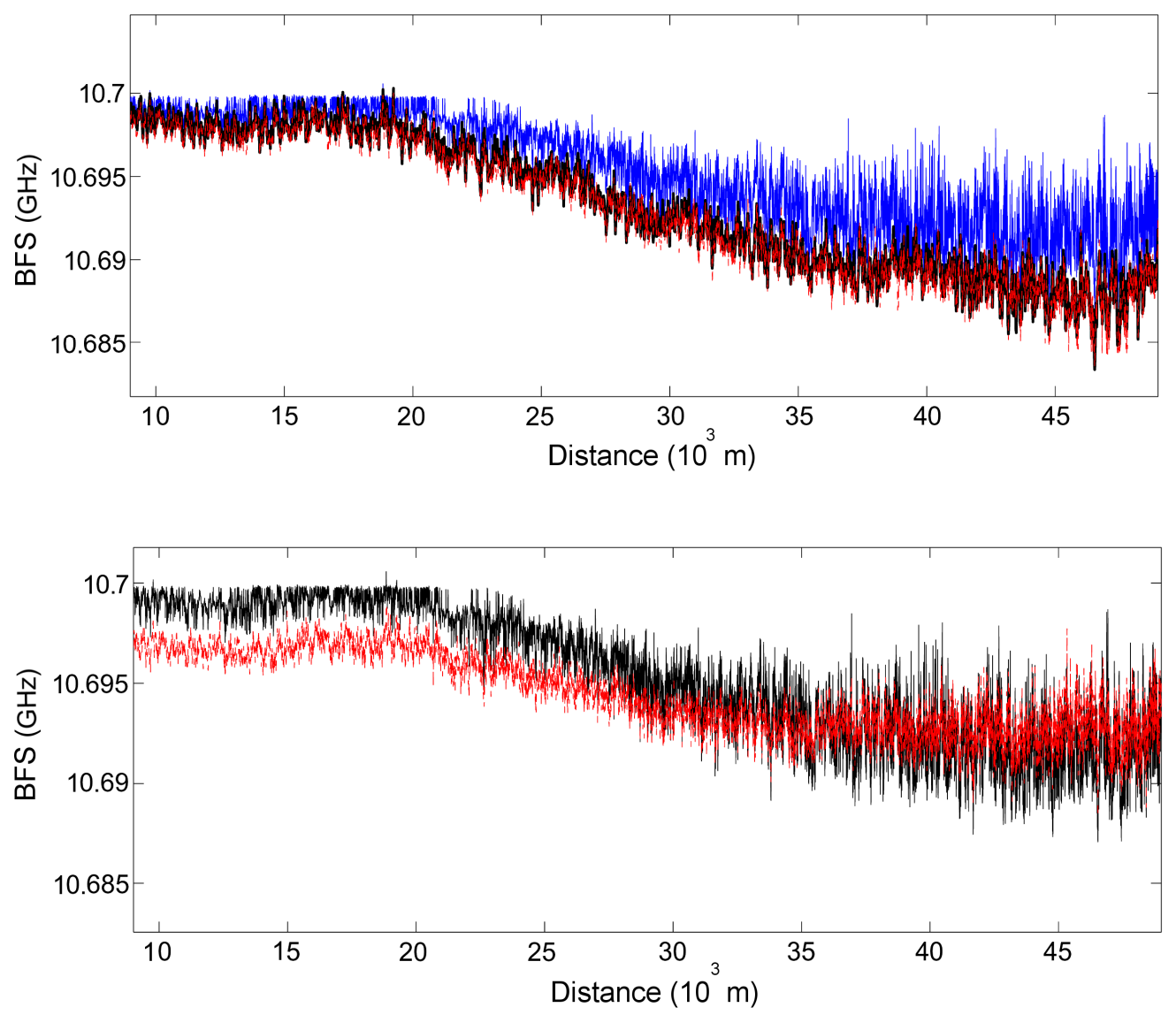

Figure 2. (a) Comparison of the BFS profiles obtained with Lorentzian fitting for the deployed fiber ( 9 to $49 \mathrm{~km})$ for different scanning granularities (2 MHz: black line; $10 \mathrm{MHz}$ : red line; $20 \mathrm{MHz}$ : blue line); (b) Comparison of the BFS profiles for the deployed fiber ( 9 to $49 \mathrm{~km}$ ) for a scanning granularity of $20 \mathrm{MHz}$ with Lorentzian fitting (black) and subpixel CDA processing (red). 


\section{CONCLUSIONS}

In this paper we have proposed the employment of subpixel algorithms to substitute the standard fitting process performed to obtain the Brillouin frequency shift in distributed BOTDA systems. The results obtained with tests developed with a conventional BOTDA system and a 50km length sensing fiber show the improved performance of this approach in some specific scenarios. BFS profiles have been obtained for different scanning granularities, giving rise to the expected degradation in the BFS estimation. For the worst case scenario, considering a $20 \mathrm{MHz}$ scenario, the CDA subpixel algorithm has exhibited a clearly improved performance in terms of signal-to-noise ratio, frequency accuracy and computational times in comparison to the Lorentzian fitting. These results suggest that the proposed solution may be of special interest for distributed dynamic measurements, where fast acquisition and processing is required. In this regard, further studies will be performed to extend this analysis to a more complete set of BOTDA scenarios.

\section{ACKNOWLEDGEMENTS}

This work has been supported by the Universidad de Cantabria and by the Spanish Ministerio de Ciencia e Innovación through project TEC2013-47264-C2-1-R.

\section{REFERENCES}

[1] D. Garus, T. Gogolla, K. Krebber et al., "Distributed sensing technique based on Brillouin optical-fiber frequency-domain analysis," Optics letters, 21(17), 1402-1404 (1996).

[2] K.-Y. Song, Z. He, and K. Hotate, "Distributed Strain Measurement with Millimeter-Order Spatial Resolution Based on Brillouin Optical Correlation Domain Analysis and Beat Lock-in Detection Scheme." ThC2.

[3] M. Nikles, L. Thevenaz, and P. Robert, "Brillouin gain spectrum characterization in single-mode optical fibers," Lightwave Technology, Journal of, 15(10), 1842-1851 (1997).

[4] R. Bernini, A. Minardo, and L. Zeni, "Dynamic strain measurement in optical fibers by stimulated Brillouin scattering," Optics letters, 34(17), 2613-2615 (2009).

[5] A. Voskoboinik, O. F. Yilmaz, A. W. Willner et al., "Sweep-free distributed Brillouin time-domain analyzer (SF-BOTDA)," Optics express, 19(26), B842-B847 (2011).

[6] J. Urricelqui, A. Zornoza, M. Sagues et al., "Dynamic BOTDA measurements based on Brillouin phase-shift and RF demodulation," Optics express, 20(24), 26942-26949 (2012).

[7] P. Bing, X. Hui-Min, X. Bo-Qin et al., "Performance of sub-pixel registration algorithms in digital image correlation," Measurement Science and Technology, 17(6), 1615 (2006).

[8] J. Alves, J. Santos, A. Carvalho et al., "Fiber Bragg sensor interrogation system based on a CCD spectrometer." 2, 909-913.

[9] J. Mirapeix, A. Cobo, C. Jaúregui et al., "Fast algorithm for spectral processing with application to on-line welding quality assurance," Measurement Science and Technology, 17(10), 2623 (2006).

[10] T. Reinsch, and J. Henninges, "Temperature-dependent characterization of optical fibres for distributed temperature sensing in hot geothermal wells," Measurement Science and Technology, 21(9), 094022 (2010).

[11] Y. Dong, P. Xu, C. Fu et al., " $1200^{\circ}$ C high-temperature distributed Brillouin optical fiber sensing based on photonics crystal fiber." 963485-963485-4. 